\title{
Lymphadenopathy due to Kikuchi-Fujimoto disease - A rare differential for a common presentation
}

\section{Claudia Wallrauch ${ }^{1}$, Tom Heller ${ }^{2}$, Bal Mukunda Dhungel ${ }^{3}$, Henry Kayera $^{1}$, Sam Phiri ${ }^{2,4,5}$, Tamiwe Monica Tomoka ${ }^{2}$}

1. Department of Internal Medicine, College of Medicine, Kamuzu Central Hospital, Lilongwe, Malawi

2. Lighthouse Clinic, Kamuzu Central Hospital, Lilongwe, Malawi

3. University of North Carolina School of Medicine, Pathology Lab, Lilongwe, Malawi

4. University of North Carolina School of Medicine, Department of Medicine, USA

5. University of Malawi, College of Medicine, School of Public Health and Family Medicine, Department of Public Health, Lilongwe, Malawi

\section{Presentation of the case}

A 22-year-old female and previously healthy patient presented at Kamuzu Central Hospital in Lilongwe, Malawi, complaining of an enlarged lymph node on the left side of her neck for 3-4 weeks in January 2017. Her case file revealed that she additionally had reported recent upper respiratory symptoms, slight fever and mild headache. No typical tuberculosis (TB) symptoms (weight loss, night sweats or cough) were present. Her Human Immunodeficiency Virus (HIV) test was negative and her full blood count was normal except for mild leukopenia. An ultrasound of the neck showed a nuchal hypoechoic, homogenous lymph node measuring 14x10 mm (Figure1).

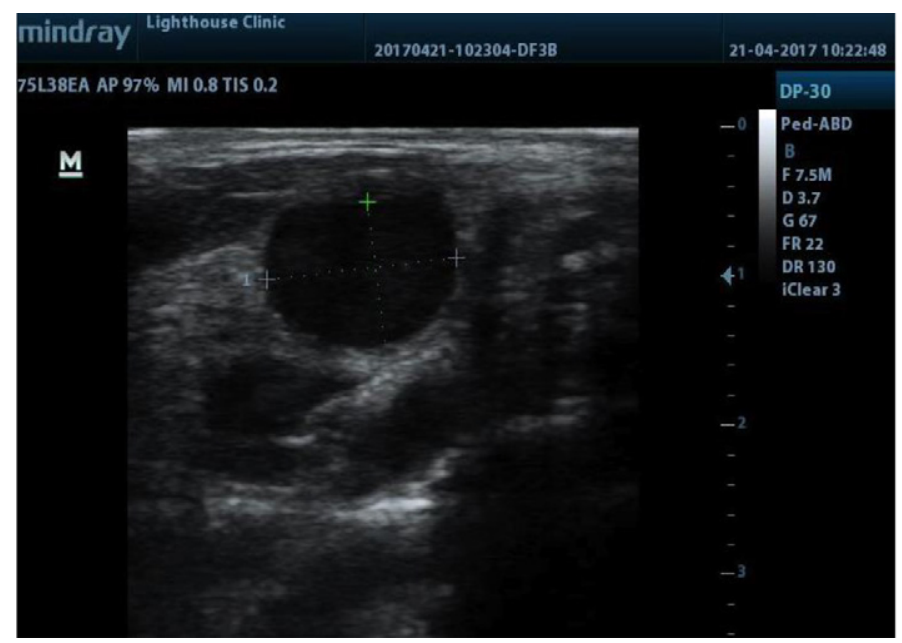

Figure 1: Ultrasound of the nuchal lymph node

As the patient was very concerned about the node and the possibility of TB and cancer, a surgical lymph node biopsy was performed. The histological examination at our laboratory showed features of necrotizing lymphadenitis compatible with Kikuchi-Fujimoto disease. The patient was reassured and treated with non-streroidal anti-inflammatory drugs (NSAIDs). The node disappeared soon but relapsed after one month. To reassure the patient, this time, a fine needle aspiration (FNA) was performed which showed, again, no sign of malignancy. Prednisolone in a low dose (20 $\mathrm{mg}$ for one week, $10 \mathrm{mg}$ for a second week and $5 \mathrm{mg}$ for a third week) was prescribed and the node disappeared. In the following months the patient stayed asymptomatic and well.

\section{Discussion}

Kikuchi-Fujimoto disease (KFD) is an enigmatic, benign disease, characterized by tender, regional lymphadenopathy predominantly in the cervical region ${ }^{1}$. It is a rare disease but known to have a worldwide distribution. The highest prevalence is seen among Asian individuals, particularly in Japan, where it was first described ${ }^{2,3}$. Only isolated cases are reported in Europe $^{1}$ and even less frequently cases are diagnosed in Africa ${ }^{4}$. KFD occurs mostly in young adults under the age of 30 with a female predominance ${ }^{5}$. The onset is acute or sub-acute, evolving over a period of two to three weeks ${ }^{5}$. Tender cervical lymphadenopathy is almost always present. Additionally, 30 to $50 \%$ of patients may have a lowgrade fever and upper respiratory symptoms ${ }^{5}$. Less frequently, weight loss, nausea, vomiting, sore throat and night sweats are described ${ }^{5}$. Although rare, in severe cases, involvement of the central nervous system and peripheral neuropathy were reported $^{5}$. KFD is typically self-limited within one to four months and the patient can thus be reassured ${ }^{6}$. Recurrence, as in our patient, is possible and reported at a rate of 3 to $5 \%{ }^{6}$. Treatment options include NSAIDs or the short-term use of steroids, mainly to relieve the distressing local and systemic complaints ${ }^{6}$.

The characteristic histological features, as seen in our case (Fig. 2a and b), are focal, well circumscribed, paracortical/ subcapsular, histiocytic necrotizing lesions with abundant karyorrhectic debris, karyolysis, scattered fibrin deposits and collections of mononuclear cells. The necrotic areas are typically devoid of neutrophils or epithelioid granulomas. Numerous palisading phagocytic histiocytes are seen around the necrosis with peripherally placed ('crescentic') nuclei. Along with these, medium-sized cells with eccentrically placed nuclei consistent with plasmacytoid dendritic cells are seen.

\section{Conclusion}

In our experience, biopsy of lymph nodes with thorough pathological work-up is a key investigation for unclear cases of lymphadenopathy and can reveal unexpected findings of "rare zebras".

Although the trigger and mechanism of cell death involved in KFD is not clear, apoptotic cell death seems to play a key role in the pathogenesis of the disease ${ }^{1}$. Some researchers suggested that KFD could be a self-limited autoimmune condition related to systemic lupus erythematous (SLE) ${ }^{1}$. 


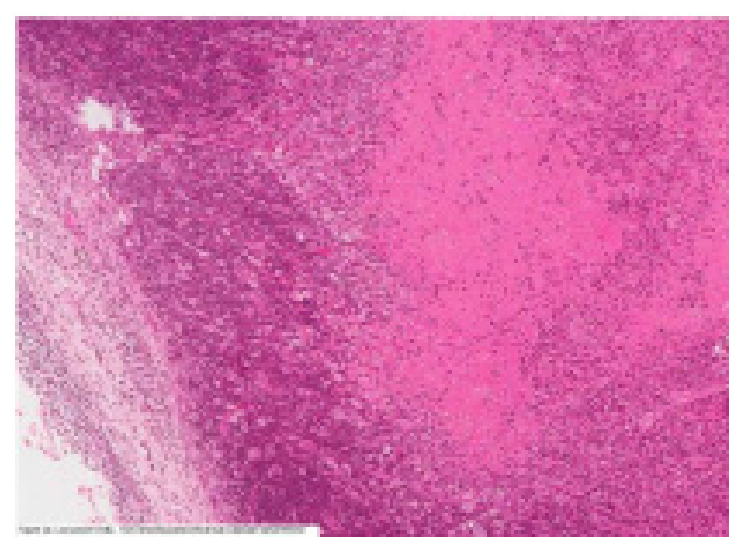

Figure 2(a): Low-power (10X) showing paracortical/sub-capsular karyorrhectic necrosis

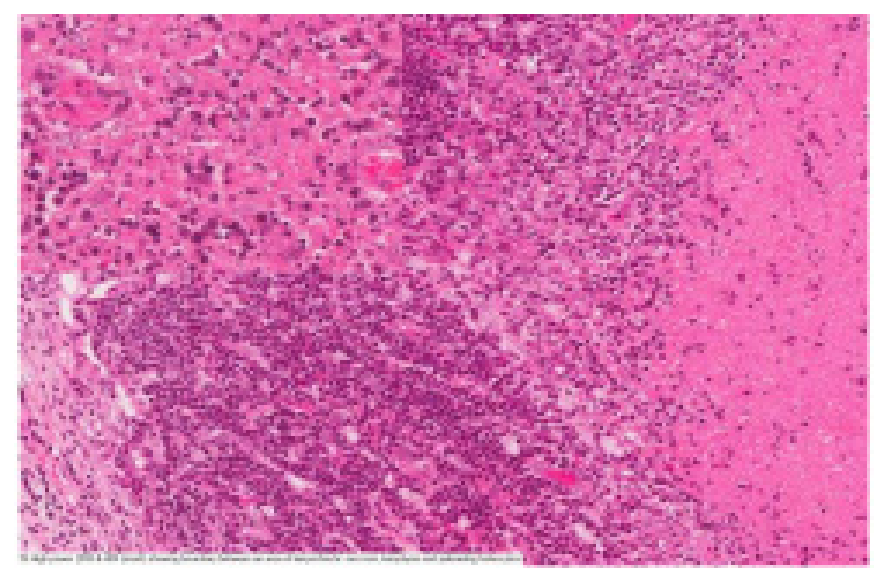

Figure 2(b): High power (20X \& 40X (inset) showing boundary between an area of karyorrhectic necrosis, karyolysis and palisading histiocytes

Infections, especially with herpes virus, have also been suggested as a possible cause ${ }^{1}$. Epstein-Barr virus (EBV), Human-Herpes Virus (HHV-6, HHV-8, parvovirus B19) are discussed in the pathogenesis but none have been proven ${ }^{1}$. In our patient, immune-stains for HHV-8, also known as Kaposi's Sarcoma Herpes Virus (KSHV), were negative.

\section{Differential diagnoses}

Enlarged lymph nodes are a frequent clinical presentation and need to be investigated according to the local most probable etiologies. In our setting with a high HIV and tuberculosis (TB) prevalence, the most common causes of lymphadenopathy are TB, lymphoma, Kaposi's sarcoma, persistent generalized lymphadenopathy and reactive lymphadenopathy due to local infections. Most of these are especially prevalent in HIV-co-infected patients. Nevertheless, other less common causes of lymphadenopathy have to be considered (Table 1) especially when the patient is HIV-negative and the clinical picture does not fit the above. KFD can practically only be diagnosed by excisional biopsy of the nodes and a substantial part of the rare number of descriptions in Africa is probably due to limited pathology services. Although it cannot be proven from our case description, KFD may be less "rare" than previously thought, if pathological services were widely available.
Table 1: Causes of lymphadenopathy

\begin{tabular}{|c|}
\hline "The Big Five" \\
\hline Tuberculosis \\
\hline Lymphoma \\
\hline Kaposi's sarcoma \\
\hline $\begin{array}{l}\text { HIV-ass. persistent generalized } \\
\text { lymphadenopathy (PGL) }\end{array}$ \\
\hline Reactive lymphadenitis in local infections \\
\hline "The Rare Zebras" \\
\hline Infectious \\
\hline Atypical mycobacteriosis \\
\hline Infectious mononucleosis (EBV) \\
\hline Brucellosis \\
\hline Tularemia \\
\hline Syphilis \\
\hline Cat-scratch disease \\
\hline Fungal infections (e.g. histoplasmosis) \\
\hline Toxoplasmosis \\
\hline Inflammatory \\
\hline Kikuchi-Fujimoto disease \\
\hline Dermatopathic lymphadenitis \\
\hline Rosai-Dorfman disease \\
\hline Langerhans cell histiocytosis \\
\hline Kimura disease \\
\hline Sarcoidosis \\
\hline Kawasaki's disease \\
\hline Systemic lupus erythematosus \\
\hline Malignant \\
\hline Metastatic carcinoma \\
\hline T-cell leukemia \\
\hline Hairy-cell leukemia \\
\hline Castleman's disease \\
\hline Myeloid tumors \\
\hline$\underline{\text { Others }}$ \\
\hline
\end{tabular}

Amyloidosis

Metabolic storage diseases

\section{Acknowledgments}

The patient and her family were informed about the plan to publish this anonymous case report and consented to its publication.

\section{Conflict of interest}

The authors have no conflict of interest to disclose.

\section{Funding}

The authors did not receive any financial support for this case report.

\section{References}

1. Bosch X, Guilabert A. Kikuchi-Fujimoto disease. Orphanet J Rare Dis 2006; 1: 18, doi:10.1186/1750-1172-1-18

2. Kikuchi M. Lymphadenitis showing focal reticulum cell hyperplasia with nuclear debris and phagocytes: a clinicopathological study. Acta Hematol Jpn. 1972; 35: 379-380. 
3. Fujimoto Y, Kozima Y, Yamaguchi K. Cervical subacute necrotizing lymphadenitis: a new clinicopathologic entity. Naika. 1972; 20: 920927.

4. Lame CA, Loum B, Fall AK, Cucherousset J, Ndiaye AR. KikuchiFujimoto disease, a rare cause of lymphadenopathy in Africa. Description of the first case in Senegal and review of the literature. Eur Ann Otorhinolaryngol Head Neck Dis 2017, 134: 347-49, http://dx.doi. org/10.1016/j.anorl.2017.02.007
5. Longaretti P, Savasta S, Caimmi D, Possenti I, Marseglia GL. KikuchiFujimoto disease complicated by peripheral neuropathy. Pediatr Neurol 2012; 46: 319-321. doi: 10.1016/j.pediatrneurol.2012.02.024

6. Dorfman RF. Histiocytic necrotizing lymphadenitis of Kikuchi and Fujimoto. Arch Pathol Lab Med. 1987; 111: 1026-1029. 